\title{
Thermo-viscoelastic modeling of the aircraft tire cornering
}

\author{
Iulian Rosu, Lama Elias-Birembaux, Frederic Lebon \\ LMA CNRS UPR 7051, Aix-Marseille University, Centrale Marseille \\ 31Chemin Joseph Aiguier, 13402 Marseille Cedex 20 France \\ rosu, elias, lebon@lma.cnrs-mrs.fr
}

Keywords: Tire, finite element, modeling, viscoelastic, heat generation

\begin{abstract}
Some numerical models are proposed for simulate the aircraft tire behaviour on the ground in critical situations. Fully coupled thermo-mechanical analysis procedures taking into account finite deformation, dynamics and frictional contact are studied; the visco-elasticity properties of the rubber were identified. A good agreement is observed at moderate speed, thus the model is extrapolated to high speeds and seems able to predict results in cases for which it is not possible to obtain realistic experimental data. In order to understand the thermal evolution of tire tread rubber materials during rolling and skidding steps, new experimental and numerical studies were also realized on tire tread rubber. The aim of this approach is to simulate and understand the effect of frictional heating on the tire behaviour.
\end{abstract}

\section{Introduction}

Landing and take-off operations are the most critical phases of an aircraft flight. Indeed, the high speed, the violent impact when the aircraft touches the ground, the need of braking efficiently, the ground irregularities, the climatic conditions (crosswind, contaminations, etc.) as well as other parameters can make these ground maneuvers very complex. Knowing at each time the forces in the contact patch allows controlling the behaviour at the tire/ground interface. For aeronautical applications, the experiments realized on real tire, at moderated speeds (less than $70 \mathrm{~km} / \mathrm{h}$ ), help to analyze the tire/ground behaviour in this reduced field. However, numerical models based of Finite Element Method, FEM are still fundamental ways to predict the tire behaviour at higher speed, taking into account the high non-linearities related to materials especially for rubber material, and coupled phenomena (contact with friction, thermal-mechanical couplings, and internal dissipation).

In this study, FEM models are proposed to simulate the aircraft tire behaviour in contact with the runway in critical situations. Fully coupled thermo-mechanical analysis procedures taking into account finite deformation, dynamics and frictional contact are studied. In the present models, the complete frictional work is converted to heat. The contact-friction state is governed by a variable friction coefficient depending on sliding velocity, contact pressure and temperature. The proposed models are compared with experimental data such as contact area, lateral and longitudinal stiffnesses, surface temperature evolution due to friction, self-alignment torque, and lateral friction coefficient. A good agreement is observed at moderate speed (less than $70 \mathrm{~km} / \mathrm{h}$ ). The present model is then extrapolated to high speeds and seems to be able to predict results in cases for which it is not possible to obtain realistic experimental data. The thermal evolution of tire tread rubber materials during rolling and skidding steps was also investigated; new experimental and numerical studies were realized on tire tread rubber specimens. The aim of the thermal approach is to simulate the frictional heating of the tire tread developed during real situations by applying an equivalent external heat flux. In the same time, the thermal diffusion through the tire tread was analyzed. 


\section{Mechanical and a fully coupled thermo-mechanical Finite element model}

In this study, mechanical and fully coupled thermo-mechanical analyses are developed to predict the friction ratio $\mu_{\mathrm{y}}$ (the ratio between the normal and the tangential force) and the self-aligning torque $\mathrm{M}_{\mathrm{z}}$ (the torque in the vertical direction). A classical rolling (CR) method based on a Lagrangian formulation was studied, thus from an observer point of view, the tire mesh (51circumferential elements) is rotating and moving forward. A variable friction coefficient $\mu(\mathrm{P}, \mathrm{T})$ depending on pressure and temperature was used in the thermo-mechanical model, real viscosity parameters were also introduced in this model. Concerning the mechanical model, a fixed friction coefficient $\mu_{0}$ was adopted. These simulations (for both models) require two numerical steps, an acceleration step from zero to a given velocity and a rolling cornering step at a constant given velocity and a given slip angle.

\section{Self aligning torque/ lateral friction coefficient}

Concerning the mechanical and thermo-mechanical analyses, our analysis is focused on grooved aircraft tires. Fig. 1 shows the evolution of the self aligning torque $\mathrm{M}_{\mathrm{z}}$ versus the slip angle. In order to show the influence of the temperature on the lateral friction coefficient $\mu_{\mathrm{y}}$ and on the self-aligning torque $\mathrm{M}_{\mathrm{z}}$, purely mechanical, and thermo-mechanical models were performed under the same loading conditions and for the same slip angles. Results were compared with experimental data. In the case of the pure mechanical simulation without any temperature dependence, we can observe that the lateral coefficient $\mu_{\mathrm{y}}$ (Fig.2) reaches a plateau equal to $\mu_{0}$ with respect to the Coulomb's friction low. $\beta_{\max }$, the angle for which the self-aligning torque is maximum, seems to be in good agreement with experimental data. The self-aligning torque $\mathrm{M}_{\mathrm{z}}$ increases up to $\beta_{\max }$ at which the full grip potential is reached. The self-aligning torque $\mathrm{M}_{\mathrm{z}}$ decreases when $\beta>\beta_{\max }$, and tends to negative values. In the case of a thermo-mechanical approach, the dependence of the lateral friction coefficient on the temperature (Fig.2) leads to a decrease in $\mu_{\mathrm{y}}$ beyond the $\beta_{\max }$ value. The selfaligning torque $\mathrm{M}_{\mathrm{z}}$ (Fig.1) falls quickly toward null values.

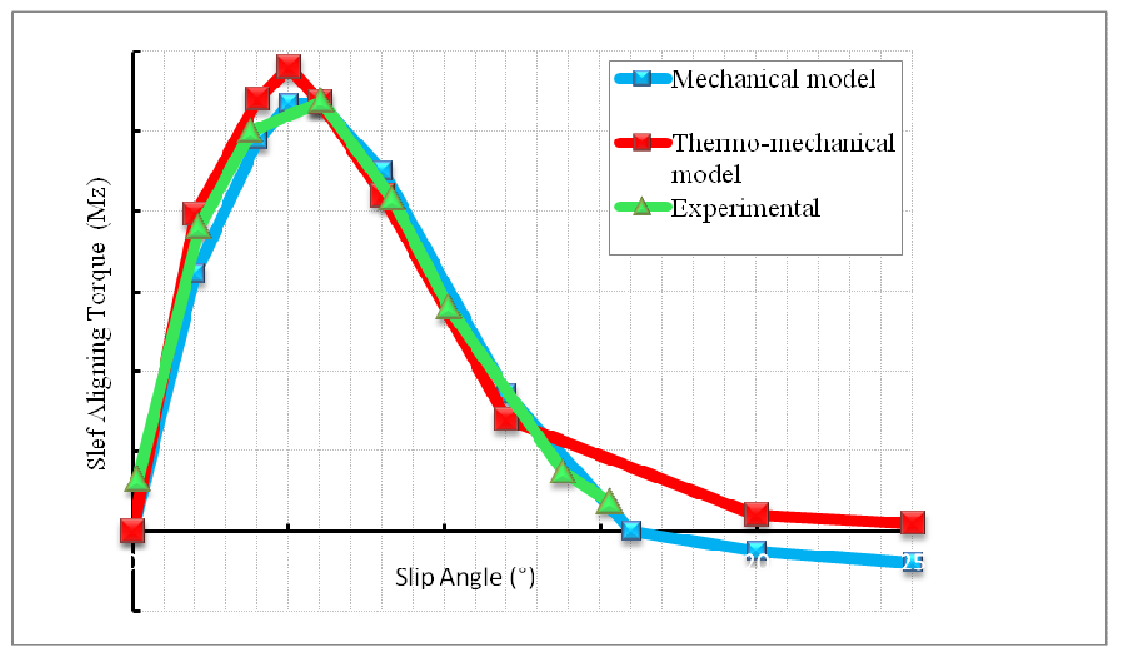

Fig. 1: Self aligning torque $\mathrm{M}_{\mathrm{z}}$, comparison between experimental data and mechanical and thermo-mechanical FE models, under given loading conditions $\left(\mathrm{F}_{\mathrm{z}}, \mathrm{p}_{0}, \mathrm{v}_{0}\right)$. 


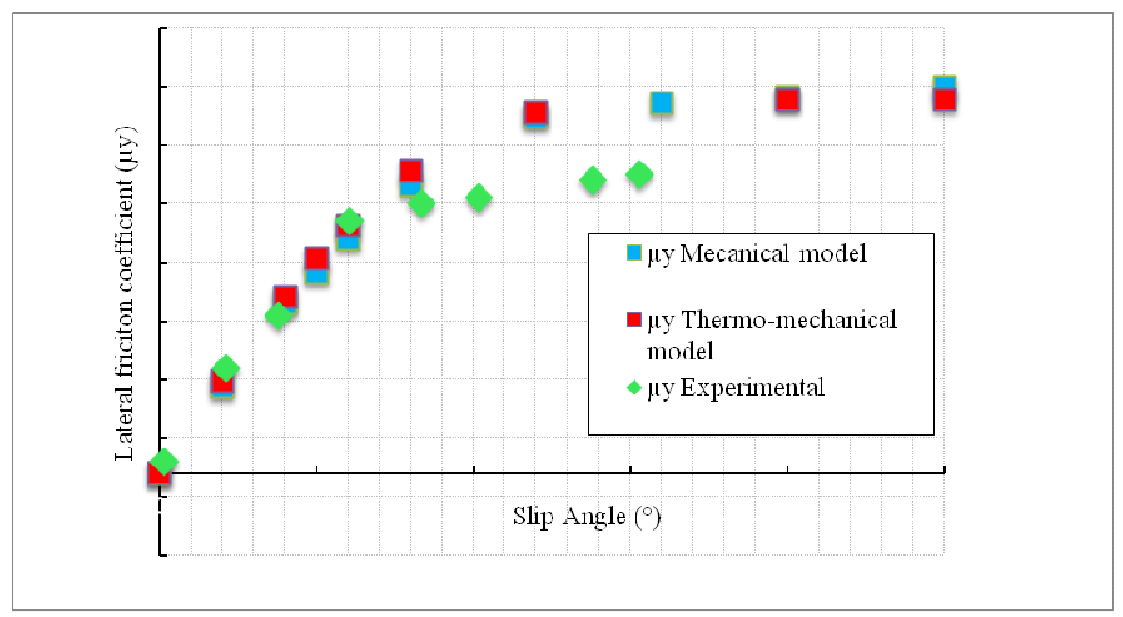

Fig. 2: Lateral friction coefficient $\mu_{\mathrm{y}}$, comparison between experimental data, mechanical and thermo-mechanical FE models, under given loading conditions $\left(\mathrm{F}_{\mathrm{z}}, \mathrm{p}_{0}, \mathrm{v}_{0}\right)$.

\section{Thermo-mechanical model, temperature evolution of an aircraft tire}

Thermo-mechanical simulation of skidding tire (tire is subjected to an inflation $\mathrm{P}_{0}$, a concentrated vertical loading $\mathrm{Fz}$, rolling velocity $\mathrm{v}_{0}$ and small slip angle $\beta_{0}$ ) was investigated; the rolling is obtained by imposing longitudinal and lateral velocities related to a slip angle. Figure 4 presents the temperature distribution in this case.

After a few seconds of rolling in cornering, an increase of the temperature occurs on tire tread and especially on groove sides; a warming zone is observed on the entire contact zone. However a slight diffusion of temperature is observed after a short period of rolling. Figure 5 presents a comparison between thermo-mechanical FEM and experimental data of a cornering simulation of an aircraft loaded tire for a given velocity $\mathrm{v}_{0}$ and for small slip angle $\beta_{0}$. In this case a good agreement is observed between the simulation and experimental data for a small angle of skidding.

In order to understand the role of a thermal barrier presented by the reinforced zones, different experimental and numerical tests were performed in laboratory tests; their purpose was to verify the evolution of the rubber behaviour during cornering rolling. These studies confirmed the necessity of taking into account the frictional heating of rubber and also internal heating produced by energy dissipation (visco-elasticity properties).

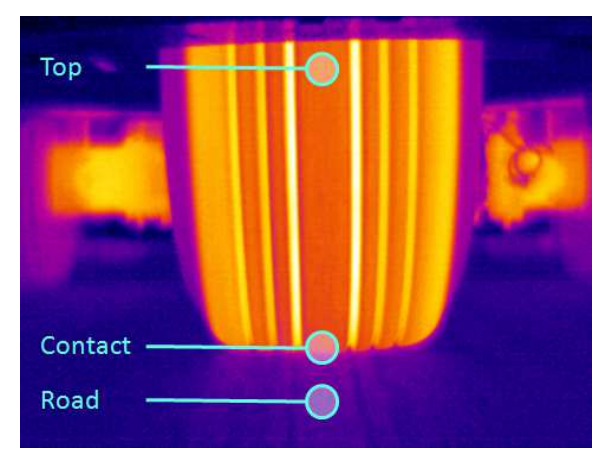

Fig. 3: Experimental measurement.

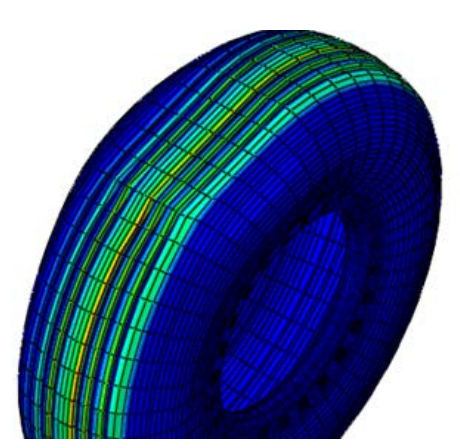

Fig. 4: FE model. 


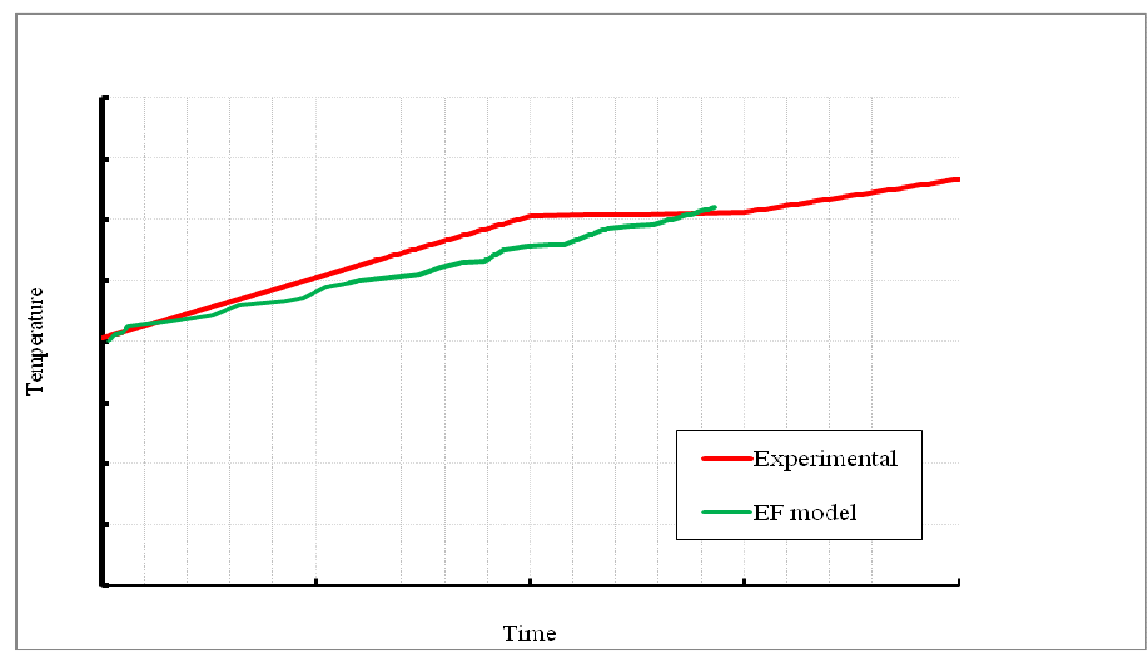

Fig. 5: Temperature evolution of tire tread, comparison between FEM and experimental data for a cornering simulation for given velocity $\mathrm{v}_{0}$ and small slip angle.

Recently we have developed a new thermo-mechanical model, able to predict the temperature evolution of skidding tire at high vertical loading and for different velocity values. Our current model was developed assuming that heat generation is governed by inelastic energy. Several experimental runs have been done on the tire for different speeds and skidding angles, temperature evolutions have been recorded by an infrared camera. Figure 6 shows temperature evolution of tread tire from experimental measurement for a given velocity $v_{1}$ and slip angle $\beta_{1}$. Figure 7 presents the first results of this new approach, comparison between experimental data and FEM model. A significant rising of temperature was observed after a short skidding time (about 0,8 second). As we mention, the thermo-mechanical FE model was developed assuming heat generation, this energy is highly depending on the stress and strain evolution during the deformation process, these in turn depend on the material viscoelastic properties of the tire. In the case of our model (fig.7) we can reasonably consider that the initial part of the temperature evolution comes from the inelastic dissipation effects while the "plateau" results of the frictional work transformed into heat flux.

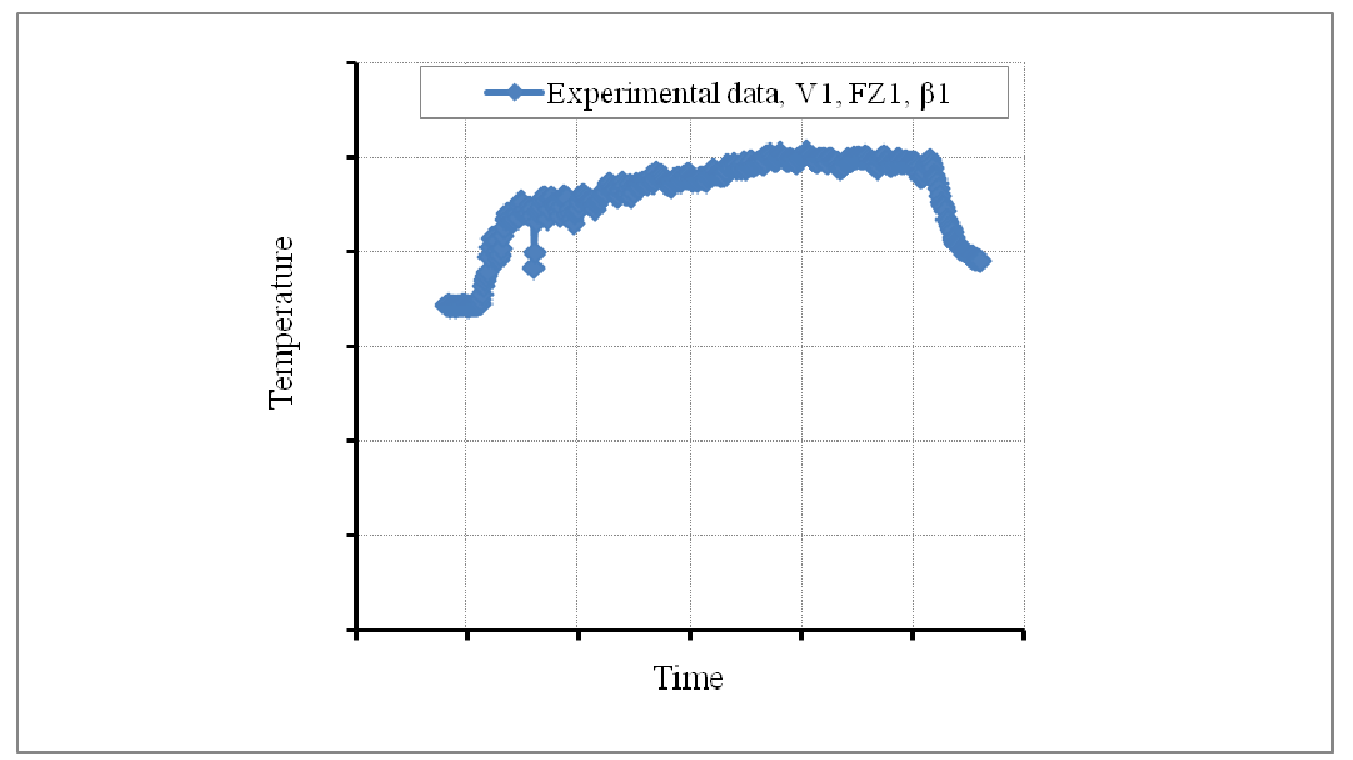

Fig. 6: Temperature evolution of tire tread, vertical loading $\mathrm{F}_{\mathrm{zl}}$, rolling velocity $\mathrm{v}_{1}$ and slip angle $\beta_{1}$. 


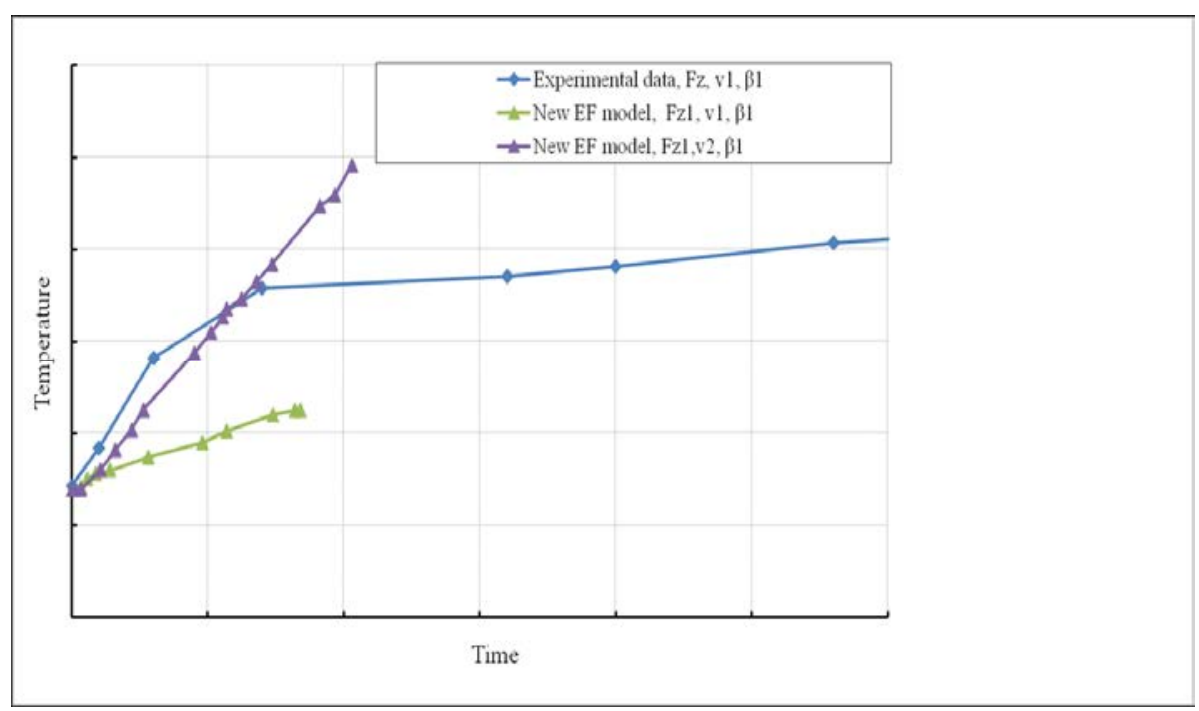

Fig. 7: Temperature evolution of tire tread, comparison between two skidding tires rolling for two different velocity $\mathrm{v}_{1}$ and $\mathrm{v}_{2}$, for a given slip angle $\beta_{1}$.

That is why we consider that an improved analysis of the viscoelastic rubber tread properties is necessary for the development of FEM model, whoever, the current model is able to predict the tire evolution at a high velocities (more than $250 \mathrm{~km} / \mathrm{h}$ ) for which it is not possible to obtain realistic experimental data (fig.7, FEM model for rolling velocity $\mathrm{v}_{2}$ ).

\section{Simulated frictional heat}

The main goal of this section is to evaluate the temperature evolution of the tire tread, by mean of a simple heat-up experiment. This study aims to simulate the frictional heating of the tire tread by applying an external heat flux and also to study the thermal diffusion through the tire tread.

\section{Experimental study}

The experimental test consists in warming-up the tire tread (fig.8) by heating flux at different temperatures $60^{\circ} \mathrm{C}$ and $100^{\circ} \mathrm{C}$ and measuring simultaneously the temperature evolution on the outer surface using two external sensors facing the tread and the groove, and inside the tire structure by means of two other infrared sensors. The heating flux was normal to the tread surface.

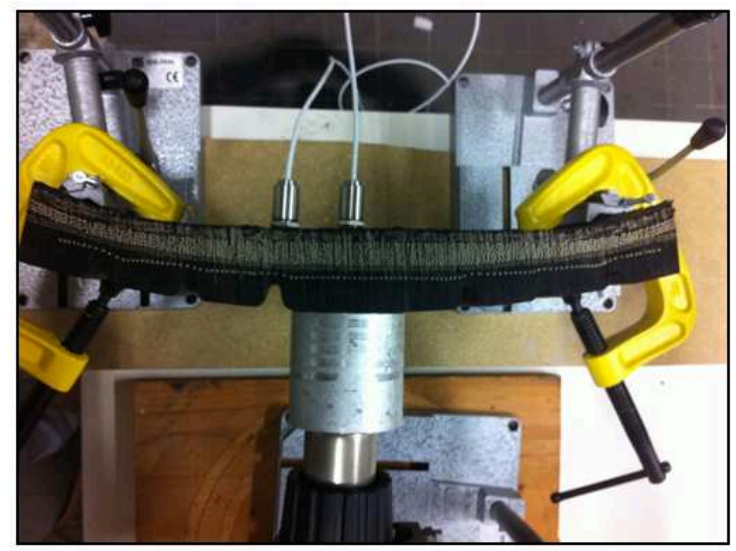

Fig. 8: Heat-up setting

Figure 9 presents the experimental analysis, as we can see, the surface temperature of tire tread rises from ambient to almost the prescribed limit within the heated area (green triangles). The surface groove temperature is almost not evolving (blue cross). For the inner evolutions corresponding to 
the heated zone, we find time shifted raises, smaller amplitudes and different slopes (blue solid). For the inner grooves the evolution profiles look very similar to the tread ones (red solid). But we can easily remark that after turning off the heating flux, while the outer tread rubber temperature starts decreasing, the groove temperature keeps rising for a while, then reaches a plateau. This proofs that the heat is "stocked" and "diffused" throughout the tire rubber and in the same time that rubber has a very small conductivity coefficient.

\section{Numerical simulation}

3D numerical model was developed to predict the thermal evolution of tread tire under heating flux. A numerical specimen identical to the physical one was created.

Figure 9 presents a numerical simulation of the tire tread rubber heated up by a given heating flux during 300 seconds. As we can observe, a good agreement is occurred between the simulated temperature of tire tread and the measured one, our present model is able to predict the temperature evolution of tread tire, and the thermal diffusion through the tire tread in a simple case of heat-up.

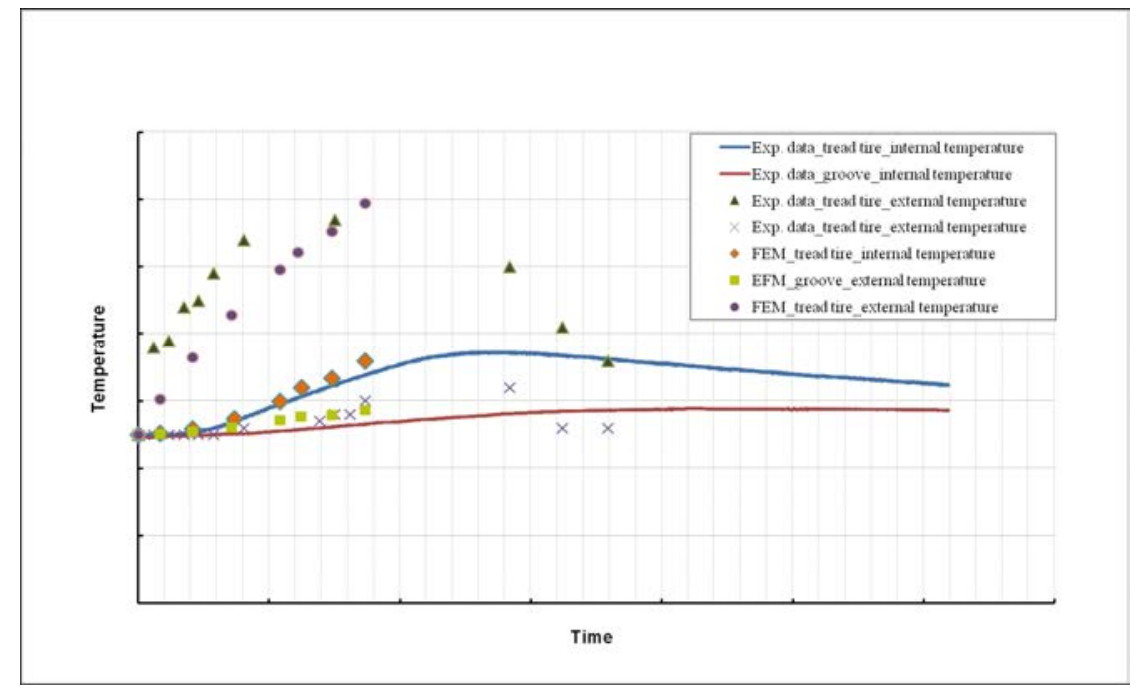

Fig. 9: Temperature evolution of external and internal layers of tread tire under heating flux, experimental and numerical results

\section{Conclusion}

In this paper, we have proposed some numerical models to simulate the aircraft tire behaviour in cornering. Fully coupled thermo-mechanical analysis procedures taking into account finite deformation were studied. The proposed models have been compared with experimental data such as a surface temperature evolution due to friction, self-alignment torque, and lateral friction coefficients. A good agreement was observed at moderate speeds (less than $70 \mathrm{~km} / \mathrm{h}$ ), the model seems to be able to predict results at high speeds, in which case it is not possible to obtain realistic experimental data.

New experimental and numerical studies were also realized on tire tread rubber. The aim of this thermal approach was to simulate and understand the effect of frictional heating on the tire rubber behaviour, a good accord was observed between experimental and numerical data. In the future, we intend to improve the models introducing more complex constitutive equations coupling thermomechanical effects, particularly taking into account viscosity and internal dissipation. Other kind of experimental devices will be also proposed in order to confirm the validity's domain of our models in various critical situations. 


\section{References}

[1] A. Kongo Kondé, Modélisation du roulement d'un pneumatique d'avion, $\mathrm{PhD}$ thesis, 2011, Aix-Marseille University.

[2] A. Kongo Kondé, I.Rosu, F. Lebon, O. Brardo, B. Devésa :Thermo-Mechanical anlysis of an aircraft tire in cornering using coupled ALE and lagrangian formulation, Central European Journal of Engineering, V3, 191-205,(2012).

[3] L. Elias-Birembaux, I. Rosu, F. Lebon: Thermo-mechanical modelling of the aircraft tyre in cornering, Machine Dynamics Research, Vol.37, No 1, 29-26(2013).

[4] L.Yeong-Jyh, H. Sheng-Jye: Temperature prediction of rolling tires by computer simulation, Taiwan Mathematics and Computers in Simulation 67, 235-249, 49(2004).

[5] A. Kongo Kondé, I. Rosu, F. Lebon, O. Brardo, B. Devésa: On the modelling of aircraft tire, Aerospace Science and Technology, 27, 1 2013, 67-75(2013).

[6] G. Heinrich, M. Klüppel, Rubber friction tread deformation and tire traction, Wear, Volume 265, Issues 7-8, 20 September, Pages 1052-1060(2008).

[7] N.D. Smith, Understanding parameters influencing tire modeling, Department of Mechanical Engineering, Colorado State University(2004). 